\title{
A Novel Hybridized Automated Manual Transmission for High Performance Cars
}

\author{
F. Vacca, G. Capilli, A. Sorniotti* \\ University of Surrey, Guildford, UK \\ *Corresponding author, e-mail: a.sorniotti@surrey.ac.uk \\ Phone: $+44(0) 1483689688$ \\ M. Fracchia, T. Remondin \\ Vocis Ltd \\ Warwick, UK
}

\author{
C. Cavallino \\ Oerlikon Graziano S.p.A. \\ Rivoli (Torino), Italy \\ F. Bottiglione \\ Politecnico di Bari \\ Bari, Italy
}

\begin{abstract}
Because of the introduction of progressively more restrictive regulations that aim to reduce fuel consumption and $\mathrm{CO}_{2}$ emissions, the automotive industry is focusing its efforts on environmentally friendly passenger cars. In particular, different electrification roadmaps are pursued by the individual car makers, and electrified drivetrain layouts are progressively introduced into the market. This process involves all vehicle segments, including high performance passenger cars. In this context, this study presents a novel hybridized automated manual transmission (HAMT) for cars with high power characteristics. The HAMT includes six gear ratios associated with the internal combustion engine, and two gear ratios associated with an electric motor. The energy efficiency of the HAMT is measured on a drivetrain test rig, and compared with that of an equivalent dual clutch transmission (DCT). Simulation results based on the experimentally measured efficiency maps show the significant energy consumption reduction of the HAMT in its internal combustion engine mode, with respect to the DCT.
\end{abstract}

Keywords-Dual clutch transmission; hybridized automated manual transmission; efficiency; fuel consumption.

\section{INTRODUCTION}

Driving comfort requirements push the automotive industry towards the generalized adoption of automatic transmissions (ATs) for internal combustion engine (ICE) driven vehicles. However, mainly because of the shear losses in the torque converter, conventional ATs have lower energy efficiency than manual layshaft transmissions [1]. For this reason, in the past decades automated manual transmissions (AMTs) were introduced, to provide automated gearshift capability with the same mechanical layout and efficiency of manual transmissions. However, AMTs provoke a wheel torque gap during gearshifts, and thus a discontinuity in the longitudinal vehicle acceleration, which represents a drivability issue. In this respect, DCTs [2] are becoming more and more popular, since they allow seamless automated gearshifts, with higher energy efficiency than conventional ATs during fixed gear operation.

In high performance passenger cars, the significant power dissipation of DCT clutch-to-clutch gearshifts requires the adoption of wet clutches, rather than dry clutches, for appropriate clutch pack cooling. Wet clutches are associated with substantial power losses, caused by: i) churning within the clutch pack; ii) clutch micro-slip; and iii) the additional cooling circuit of the wet clutch pack [3]. i)-iii) represent major obstacles to the energy-efficient operation of DCTs in high performance vehicles.

In parallel, the ongoing electrification process has led to the introduction of hybridized drivetrain layouts including electric machines (EMs), acting as motors or generators depending on the operating condition of the vehicle. With respect to the position of the EM within the hybrid electric drivetrain, different configurations can be identified (see Fig. 1): a) P0, with the EM acting as a belt driven starter/generator; b) P1, with the EM connected to the ICE crankshaft; c) P2, with the EM mounted before or after the clutch; d) P3, with the EM connected to the gearbox (GB in Fig. 1) secondary shaft; and e) $\mathrm{P} 4$, with the EM installed on the axle [4]. $\mathrm{K} 0$ is used to disconnect the crankshaft so that the drag associated to the ICE rotation is avoided. Each configuration offers different possibilities. For example, in the P0, P1 and P2 configurations the EM can support the ICE while taking advantage of the transmission gear ratio. In the $\mathrm{P} 3$ and $\mathrm{P} 4$ configurations appropriate EM torque control can provide torque gap compensation during gearshift actuation in case of singleclutch transmissions [5].

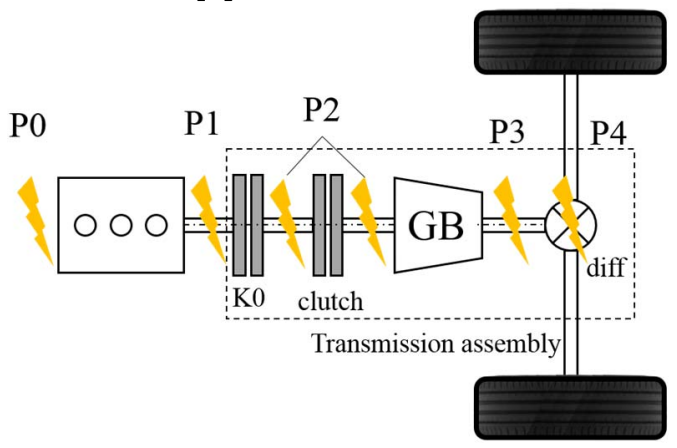

Fig. 1. Hybridization layouts.

In this context, this study describes a novel hybridized AMT layout, referred to as HAMT in the remainder, aimed at substituting a production 7-speed DCT with wet clutches for high performance passenger cars, i.e., with maximum power values in excess of $400 \mathrm{~kW}$. The experimentally measured 
efficiency maps of the two transmissions are compared. The performance of the electric part of the HAMT is analyzed by means of steady-state efficiency tests. A simulation model is used to assess the energy consumption performance of the DCT and HAMT in ICE mode, and to demonstrate the potential energy consumption reduction of the HAMT.

The paper is organized as follows. Section II describes the case study transmission layouts. Section III deals with the experimental test procedures for measuring the efficiency characteristics of the specific DCT and HAMT, while Section IV discusses the experimental efficiency maps. Section V presents the simulation model and fuel consumption results for the DCT and the ICE mode of the HAMT. Finally, Section VI summarizes the main conclusions.

\section{THE CASE STUDY TRANSMISSIONS}

The schematics of the case study DCT and HAMT are shown in Fig. 2 and Fig. 3, respectively. The two transmissions target the same category of high-performance rear-wheel-drive passenger cars, with a front longitudinal internal combustion engine connected by a driveshaft to the clutch and transmission located on the rear axle.

In Fig. 2 the DCT [6] is represented as the combination of two sub-systems, each of them including: i) one of the two coaxial wet clutches; ii) one of the two parallel co-axial primary shafts; and iii) the respective secondary shaft. Wet clutches rather than dry clutches - are used for vehicle applications with high values of maximum ICE torque, such as the one of this study. As mentioned in Section I, this provokes additional power dissipation, with respect to the more conventional case of dry clutches. The inner input shaft carries the odd gears (I, III, V and VII), which are selected through the synchronizers s1 and s2. The outer input shaft carries the three even gears (II, IV and VI) and the reverse gear (R), which are selected through the synchronizers s3 and s4. The two secondary shafts are connected to the same output shaft, which transmits the torque to the open differential through the final reduction ratio.

The HAMT [7, 8] includes six gears that are associated with the ICE, and two gears that are associated with the EM. The choice of six ICE gear ratios in the HAMT, rather than the seven gear ratios of the DCT, is the consequence of a vehicle simulation based design process of the transmission, aimed at achieving mechanical simplification and high efficiency, without compromising the longitudinal acceleration and fuel consumption. The ICE-related gears are characterized by the typical set-up of conventional AMTs, with hydraulically controlled synchronizers (s1, s2 and s3 in Fig. 3) and a dry clutch. On the EM side, the first gear, indicated as I EM in the remainder, is obtained by connecting the EM with a planetary gear set, $\mathrm{PG}$, through a controllable EM gear selector. This is followed by a sequence of two gear drops, drop1 and drop2 in Fig. 3, and a hybrid coupling, HC, implemented through a controllable dog clutch, connecting the transmission output shaft with the third gear on the ICE side of the HAMT. The second EM gear, indicated as II EM, is achieved with a dog clutch that connects the EM directly to the sequence of two gear drops and the HC. As the EM speed can be controlled in closed-loop through the modulation of the EM torque, a synchronizer is not needed for the EM gearshifts.

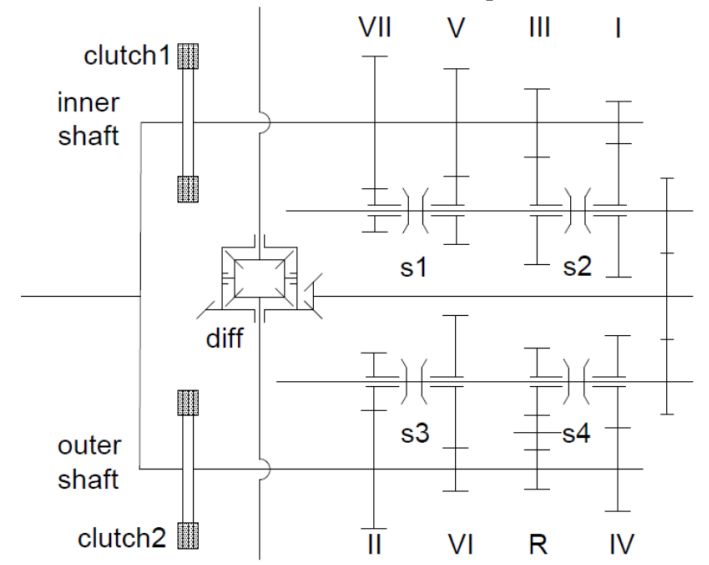

Fig. 2. DCT mechanical layout.

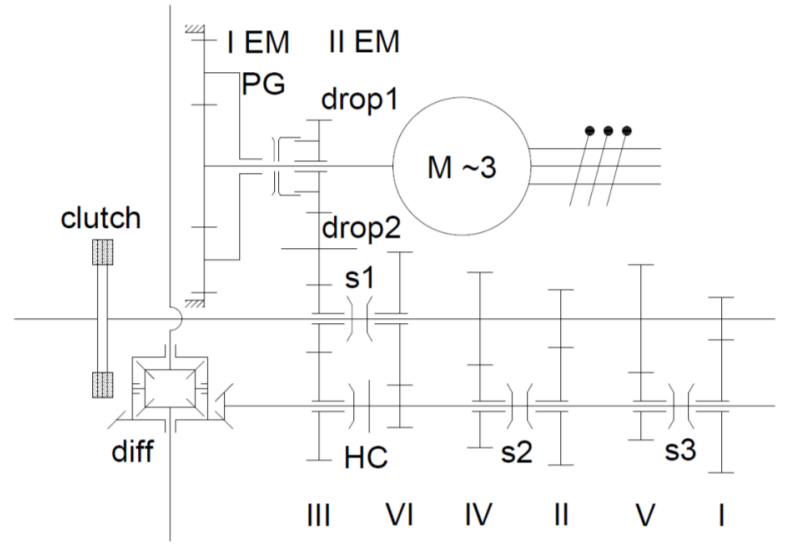

Fig. 3. HAMT mechanical layout.

TABLE I. HAMT OPERATING MODES AND THE CORRESPONDING STATUS OF EACH ACTUATOR (ADAPTED FROM [3]).

\begin{tabular}{|c|c|c|c|c|c|c|}
\hline Mode & $\begin{array}{c}\text { EM } \\
\text { status }\end{array}$ & $\begin{array}{c}\text { ICE } \\
\text { status }\end{array}$ & $\begin{array}{l}\text { EM } \\
\text { gear } \\
\end{array}$ & $\begin{array}{l}\text { ICE } \\
\text { gear } \\
\end{array}$ & $\begin{array}{c}\text { Hybrid } \\
\text { coupling }\end{array}$ & $\begin{array}{c}\text { Dry } \\
\text { clutch }\end{array}$ \\
\hline ICE & OFF & ON & $\mathrm{N}$ & I-VI & Engaged & Closed \\
\hline $\begin{array}{c}\text { Fully } \\
\text { electric (P3) }\end{array}$ & ON & OFF & I & $\mathrm{N}$ & Engaged & Closed \\
\hline Hybrid electric & ON & ON & I or II & I-VI & Engaged & Closed \\
\hline $\begin{array}{c}\text { Reverse } \\
\text { Gear }\end{array}$ & ON & OFF & I & I & Engaged & Open \\
\hline $\begin{array}{c}\text { Cranking from } \\
\text { stop and recharge }\end{array}$ & ON & Cranking & I & III & Disengaged & Closed \\
\hline $\begin{array}{c}\text { Cranking from } \\
\text { fully electric mode }\end{array}$ & $\mathrm{ON}$ & Cranking & I & III-VI & Engaged & Closing \\
\hline
\end{tabular}

The operating modes of the HAMT are listed in Table I, which indicates the status of the ICE and EM, as well as the condition of the EM gear selector, ICE synchronizers, HC and dry clutch. In fully electric mode the vehicle operates at low-tomedium speeds with the EM, and the sole I EM gear is normally allowed. On the contrary, in hybrid mode both the I EM and II EM gears can be selected, since this mode is associated with the whole range of vehicle speeds.

In fully electric mode the HAMT could work in either P2 or P3 configurations (Fig. 1), depending on the status of s1:

- In P2 the III ICE gear and the HC are engaged, and 
therefore the EM is connected to the primary shaft. Hence, the P2 configuration can be used for cranking the ICE during the transitions from the fully electric mode to the hybrid or ICE modes.

- In P3 (this is the fully electric configuration reported in Table I) the III ICE gear is disengaged, the HC is engaged, and therefore the EM is connected to the secondary shaft. The III ICE gear is used as a drop, hence the primary shaft does not take part to the motion transmission. The clutch can be closed as the input shaft does not rotate.

Table I does not include the description of the gearshift phases. In particular, during the ICE gearshifts in ICE mode or hybrid electric mode, the EM gear selector, the $\mathrm{HC}$ and the EM torque are controlled to compensate the wheel torque gap caused by the disengagement and re-engagement of the clutch. The EM torque-fill operation allows the achievement of good drivability during gearshifts with a single clutch. The ICE gearshift actuation with a single clutch brings a significant reduction of the clutch slip power losses - and thus the clutch cooling requirements - during the disengagement and reengagement phases, with respect to the clutch-to-clutch gearshifts of the DCT. This allows the adoption of a single dry clutch also for high performance vehicles.

\section{EXPERIMENTAL SET-UP}

The experimental tests of the case study DCT and HAMT were executed on the transmission test rig of the University of Surrey (Fig. 4). The test rig includes: i) an input motor, which emulates the ICE; ii) two output motors, i.e., the right and left motors in Fig. 4, which emulate the road load; iii) a cooling/heating system for controlling the transmission oil temperature and the HAMT EM coolant temperature; iv) the DC power supply for the HAMT EM; and v) a dSPACE system for data acquisition, real-time simulation and control.

The test piece consists of: i) the transmission, i.e., the DCT or the HAMT, including clutch, gearbox and differential; ii) the EM with the respective inverter (if applicable); iii) the halfshafts; and iv) the transmission control unit (TCU). The DCT was tested with the production micro-slip controller of its clutches, to ensure that the measurements are representative of the real operating conditions.

The steady-state efficiency measurement procedure follows the general guidelines in [9]:

1. The oil temperature is set to the target value by using a consistent heating/cooling procedure.

2. The relevant gears are selected.

3. The relevant transmission clutch is engaged.

4. The output motor speeds are set to the target value.

5. The input motor torque or the HAMT EM torque is applied in steps, to cover the designed range of measurement points. For each point the torque is kept constant for $30 \mathrm{~s}$, once the steady-state condition is reached.

6. The input torque is gradually removed.

7. The output motor speed is gradually reduced.

The procedure was repeated for all gears, pre-determined speed values, and two transmission oil temperatures, i.e., $30{ }^{\circ} \mathrm{C}$ and $70^{\circ} \mathrm{C}$.
For each set point, in traction the instantaneous output power, $P_{\text {out }}$, is:

$$
\begin{aligned}
P_{\text {out }}(t)=T_{\text {out }, \text { left }}(t) \omega_{\text {out, left }}(t) \\
+
\end{aligned}
$$

where $t$ is time; $T_{\text {out,left }}$ and $T_{\text {out,right }}$ are the left and right hub torques; $\omega_{\text {out,left }}$ and $\omega_{\text {out,right }}$ are the left and right hub speeds. In case of DCT testing and HAMT ICE mode testing, the instantaneous input power, $P_{i n}(t)$, is:

$P_{\text {in }}(t)=T_{\text {in }}(t) \omega_{\text {in }}(t)$

where $T_{\text {in }}$ is the input torque, and $\omega_{\text {in }}$ is the input motor speed. In the fully electric mode of the HAMT, in traction $P_{i n}(t)$ is:

$P_{\text {in }}(t)=P_{\text {inv }}(t)=I_{\text {inv }}(t) V_{\text {inv }}(t)$

where $P_{i n v}$ is the HAMT inverter input power; $I_{i n v}$ is the HAMT inverter input current; and $V_{i n}$ is the HAMT inverter input voltage. In regeneration the expressions in Eq. (1) and Eq. (3) swap with each other. The transmission or electric drivetrain efficiency, $\eta_{t r / D T}$, is calculated as the ratio between the average output power, $\bar{P}_{\text {out }}$, and the average input power, $\bar{P}_{i n}$, during the measurement interval:

$\eta_{t r / D T}=\frac{\bar{P}_{\text {out }}}{\bar{P}_{\text {in }}}=\frac{\frac{1}{N} \sum_{t=1}^{N} P_{\text {out }}(t)}{\frac{1}{N} \sum_{t=1}^{N} P_{\text {in }}(t)}$

where $N$ is the number of values in the $30 \mathrm{~s}$ measurement interval.

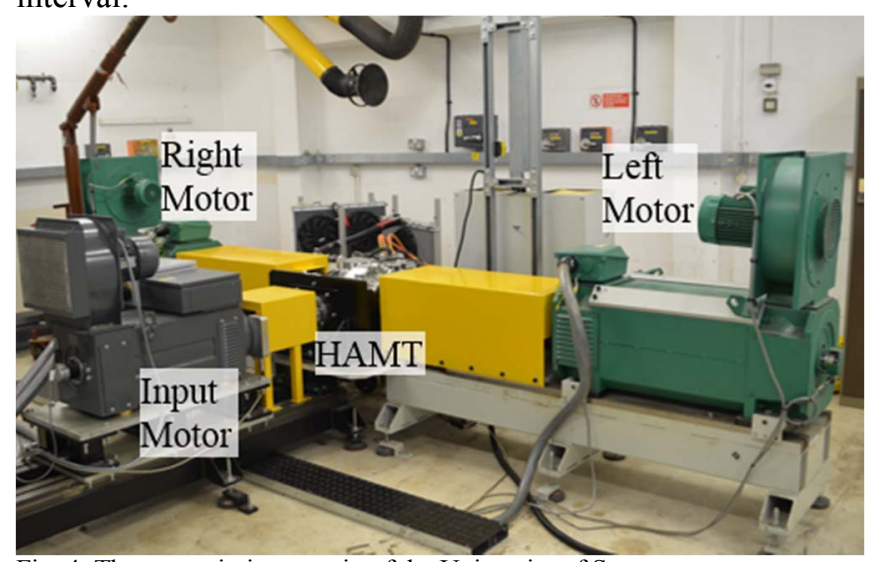

Fig. 4. The transmission test rig of the University of Surrey.

\section{EXPERIMENTAL RESULTS}

This section reports a selection of the steady-state efficiency measurements. In particular, Section IV.A shows the comparison between the DCT and the HAMT in its ICE mode, while Section IV.B discusses the HAMT efficiency in fully electric mode.

\section{A. DCT and HAMT: steady-state efficiency}

Fig. 5 is a sample of the DCT and HAMT efficiency measurement results in the different gears, i.e., I-VII for the DCT, and I-VI for the HAMT in its ICE mode, at $\omega_{\text {in }}=1000$ $\mathrm{rpm}$ and $\omega_{\text {in }}=2000 \mathrm{rpm}$, and $30^{\circ} \mathrm{C}$ of lubricant temperature.

The two transmissions show similar trends, i.e.: i) their efficiency is an increasing function of $T_{i n}$; ii) their efficiency 
decreases with $\omega_{\text {in }}$; and iii) the first gear is the most efficient transmission ratio. However, the HAMT exhibits consistently higher efficiency values, with less significant variations with $T_{i n}$. For example, in I gear at $T_{i n}=160 \mathrm{Nm}$ and $\omega_{\text {in }}=1000$ $\mathrm{rpm}, \eta_{t r}$ is $\sim 87 \%$ for the DCT (Fig. $6 \mathrm{a}$ ), and $\sim 96 \%$ for the HAMT (Fig. 6c). In general, at $30^{\circ} \mathrm{C}$ the efficiency benefit of the HAMT, expressed as $\triangle \eta_{H A M T-D C T}=100\left(\eta_{H A M T}-\right.$ $\left.\eta_{D C T}\right) / \eta_{D C T}$, ranges between $\sim 9 \%$ and $\sim 13 \%$ for input torque values $>100 \mathrm{Nm}$. For lower torque values, which are typical of conventional driving cycles, the difference is even larger. Similar trends of the efficiency characteristics were measured at $70{ }^{\circ} \mathrm{C}$, with a reduced efficiency difference among DCT and HAMT, with $\triangle \eta_{H A M T-D C T}$ ranging between $\sim 5 \%$ and $\sim 9 \%$ for medium-high torque values.
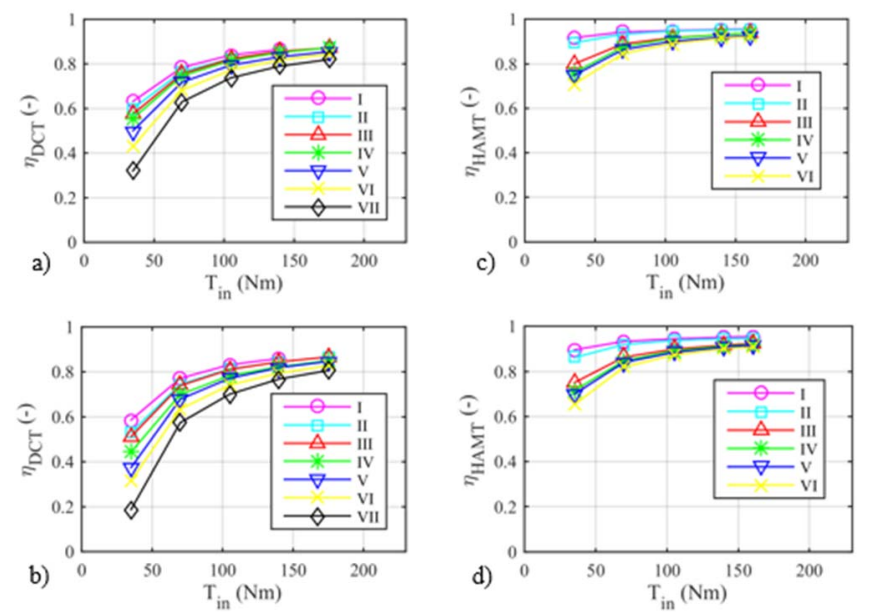

Fig. 5. Transmission efficiency measurements at $30^{\circ} \mathrm{C}$ : a) DCT at $\omega_{\text {in }}=1000$ rpm; b) DCT at $\omega_{i n}=2000 \mathrm{rpm}$; c) HAMT at $\omega_{\text {in }}=1000 \mathrm{rpm}$; d) HAMT at $\omega_{\text {in }}=2000 \mathrm{rpm}$.

\section{B. HAMT: electric drivetrain efficiency}

The P2 and P3 configurations of the fully electric mode of the HAMT are compared in terms of steady-state efficiency. The electric drivetrain efficiencies, $\eta_{D T, P 2}$ and $\eta_{D T, P 3}$, include the contributions of the inverter, EM, transmission, half-shafts and constant velocity joints. Fig. 6 is an extract of the experimentally measured maps in traction, at $\omega_{E M}=2000 \mathrm{rpm}$ and $\omega_{E M}=5000 \mathrm{rpm}$, where $\omega_{E M}$ is the EM speed. $\eta_{D T, P 2}$ and $\eta_{D T, P 3}$ increase with the EM torque, $T_{i n}$, while there is not a general trend with respect to speed. The efficiencies of the P2 and P3 configurations are very close to each other in the I EM gear at $2000 \mathrm{rpm}$, i.e., they differ by less than 1\%. This suggests that at low vehicle speeds $(\sim 20 \mathrm{~km} / \mathrm{h})$ there would not be any substantial energy consumption difference among the two configurations. In the I EM gear at $\omega_{E M}=5000 \mathrm{rpm}(\sim 50$ $\mathrm{km} / \mathrm{h}$ ), the efficiencies of P2 and P3 are close to each other at medium-high torque values, e.g., at $T_{\text {in }}=90 \mathrm{Nm}$, while $\Delta \eta_{P 3-P 2}=100\left(\eta_{D T, P 3}-\eta_{D T, P 2}\right) / \eta_{D T, P 3}$ is significant at low $T_{\text {in }}$, i.e., it is $\sim 6 \%$ at $15 \mathrm{Nm}$. The results for the II EM gear, included for completeness, show: i) lower efficiency for both P2 and P3 than in the I EM gear; and ii) a wider difference between $\mathrm{P} 2$ and $\mathrm{P} 3$, where the latter is more efficient especially at high speed, because of the reduced number of rotating components.

Fig. 7 is the efficiency comparison between traction and regeneration, in the HAMT P3 configuration. For the investigated tests, the HAMT is more efficient in traction at $\omega_{E M}=2000 \mathrm{rpm}$, where the difference of the efficiency values, $\eta_{D T, \text { Traction }}-\eta_{D T, \text { Regeneration }}$, ranges from $\sim 3$ to $\sim 4 \%$. At $\omega_{E M}=5000 \mathrm{rpm}$ the efficiencies are approximately the same.
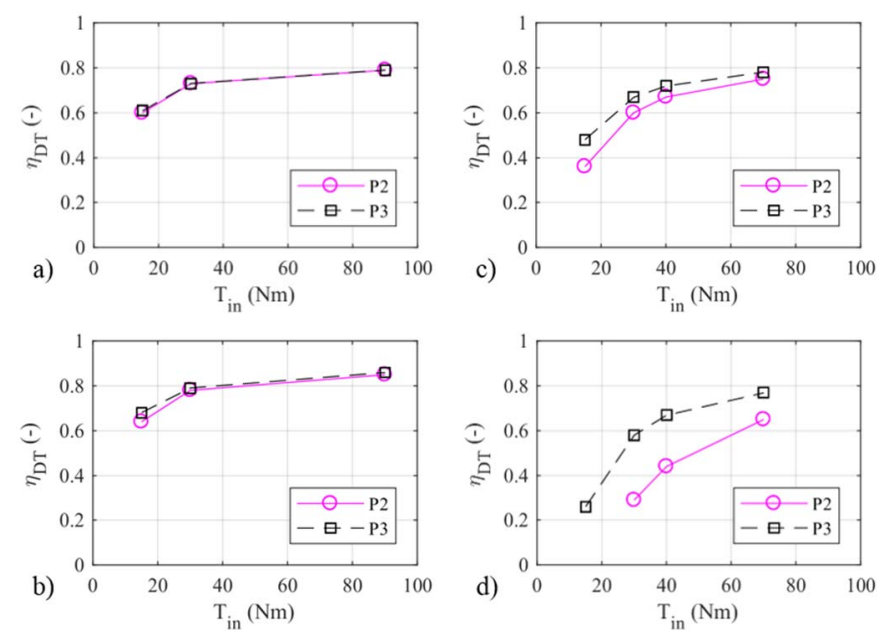

Fig. 6. HAMT efficiency measurements in fully electric mode at $70{ }^{\circ} \mathrm{C}$; comparison of P2 and P3 configurations: a) I EM gear at $\omega_{E M}=2000 \mathrm{rpm}$; b) I EM gear at $\omega_{E M}=5000 \mathrm{rpm}$; c) II EM gear at $\omega_{E M}=2000 \mathrm{rpm}$; d) II EM gear at $\omega_{E M}=5000 \mathrm{rpm}$.
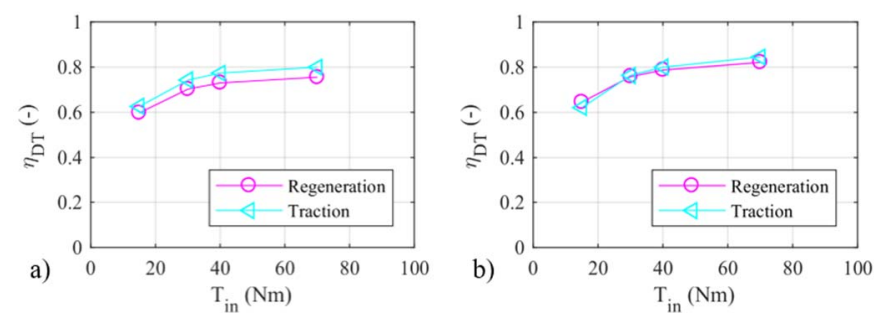

Fig. 7. HAMT efficiency measurements in fully electric mode (P3 configuration) in traction and regeneration at $30{ }^{\circ} \mathrm{C}$ : a) I EM gear at $\omega_{E M}=$ $2000 \mathrm{rpm}$; b) I EM gear at $\omega_{E M}=5000 \mathrm{rpm}$.

\section{FUEL CONSUMPTION ALONG DRIVING CYCLES}

This section uses vehicle simulations to assess the fuel consumption reduction achievable through the HAMT in its ICE mode, with respect to the same vehicle with the production DCT. This analysis does not consider the further potential benefits associated with the hybrid mode of the HAMT, which will be the subject of future studies.

\section{A. Simulation model}

A validated Matlab/Simulink backward facing simulation model was implemented to estimate the vehicle fuel consumption along a selection of driving cycles.

The main assumptions are: i) the reference vehicle speed, $V_{v e h}$, can be continuously achieved, which is verified for the driving cycles of this study and the adopted vehicle parameters; ii) tire slip is neglected, which is a reasonable approximation in the normal driving conditions typical of standardized driving cycles, see also the specific power loss studies in $[10,11]$; and iii) the gearshifts occur instantaneously without energy dissipations. Further details of the actual gearshift process for 
the two transmissions are provided in [7] and [8], where the latter includes a gearshift controller for the minimization of the clutch energy dissipation.

$V_{v e h}$, defined in accordance with the selected driving cycle, is used to calculate the required drivetrain torque at the wheel, $T_{D T}$ :

$$
\begin{aligned}
T_{D T}=R_{w}\left(\frac{1}{2} \rho C_{d r a g} S V_{v e h}^{2}\right. \\
+\tanh \left(k_{\text {hyper }} V_{\text {veh }}\right)\left(f_{0}+f_{2} V_{\text {veh }}^{2}\right) m g \\
\left.+m_{\text {app }} \frac{d V_{\text {veh }}}{d t}\right)
\end{aligned}
$$

where $R_{w}$ is the wheel radius; $\rho$ is air density; $C_{d r a g}$ is the aerodynamic drag coefficient; $S$ is the frontal area of the vehicle; $f_{0}$ and $f_{2}$ are the tire rolling resistance coefficients; $m$ is the vehicle mass; and $g$ is gravity. The hyperbolic tangent function, $\tanh \left(k_{\text {hyper }} V_{v e h}\right)$, is used to eliminate the tire rolling resistance contribution during the zero-speed phases of the driving schedules. The apparent vehicle mass, $m_{a p p}$, accounts for the mass moment of inertia of the main rotating parts of the drivetrain in the selected gear:

$m_{\text {app }}=m+\frac{4 J_{w}}{R_{w}^{2}}+\frac{J_{I C E}+J_{\text {clutch }}}{R_{w}^{2}} i_{k, I C E}^{2}$

where $J_{w}$ is the wheel mass moment of inertia; $J_{I C E}$ is the mass moment of inertia of the moving parts of the ICE; $J_{\text {clutch }}$ is the mass moment of inertia of the rotating components of the clutch assembly; and $i_{k, I C E}$ is the value of the overall gear ratio, from the engine to the wheels, in the selected gear $k$.

By considering the engaged wet clutch micro-slip, which is relevant to the sole DCT, in traction the angular speed of the ICE, $\omega_{I C E}$, is:

$\omega_{I C E}=\omega_{w} i_{k, I C E}+\Delta \omega_{M S}=\frac{V_{v e h}}{R_{w}} i_{k, I C E}+\Delta \omega_{M S}$

where $\omega_{w}$ is wheel speed, and $\Delta \omega_{M S}$ is the speed difference corresponding to the wet clutch micro-slip, which is zero in the HAMT. The ICE torque, $T_{I C E}$, is estimated by equating the power at the transmission primary shaft, $T_{I C E}\left(\omega_{I C E}-\right.$ $\left.\Delta \omega_{M S}\right)=T_{I C E} \omega_{w} i_{k, I C E}$, to the sum of: i) the required drivetrain power, $P_{D T}=T_{D T} \omega_{w}=T_{D T} V_{v e h} / R_{w}$; and ii) the transmission power losses excluding the wet clutch micro-slip effect (if relevant to the specific transmission), $P_{\text {loss }, w / o m s} . P_{\text {loss }, w / o m s}$ is calculated from the experimentally measured transmission efficiency maps, discussed in Section IV, and is expressed as a function of transmission torque, speed, temperature and selected gear. The transmission system model has the option of including a simplified thermal model based on a lumped parameter approach. In summary, $T_{I C E}$ is expressed as:

$T_{I C E}=\frac{P_{D T}+P_{\text {loss }, w / o M s}}{\frac{V_{v e h}}{R_{w}} i_{k, I C E}}$

An ICE brake specific fuel consumption map, $b_{I C E}\left(\omega_{I C E}, T_{I C E}, \theta_{I C E}\right)$, where $\theta_{I C E}$ is the ICE coolant temperature, was measured during ICE tests carried out by the industrial partners of the project. An extract of the map is reported in Fig. 8. This is used by the model for fuel consumption estimation. The fuel mass flow rate, $\dot{m}_{I C E}$, and volume consumption per unit distance, $V_{I C E, d}$, are computed from $b_{I C E}$ and the engine power demand, $P_{I C E}=T_{I C E} \omega_{I C E}$ :

$$
\left\{\begin{array}{c}
\dot{m}_{I C E}=b_{I C E}\left(\omega_{I C E}, T_{I C E}\right) P_{I C E} \\
V_{I C E, d}=\frac{\dot{m}_{I C E}}{\rho_{\text {fuel }} V_{\text {veh }}}
\end{array}\right.
$$

where $\rho_{\text {fuel }}$ is fuel density. From $V_{I C E, d}$, the $\mathrm{CO}_{2}$ emissions can be estimated with the appropriate conversion factor, i.e., $\sim 2.35$ $\mathrm{kg}$ of $\mathrm{CO}_{2}$ per liter of consumed petrol, according to the analysis and recommendations in [12].

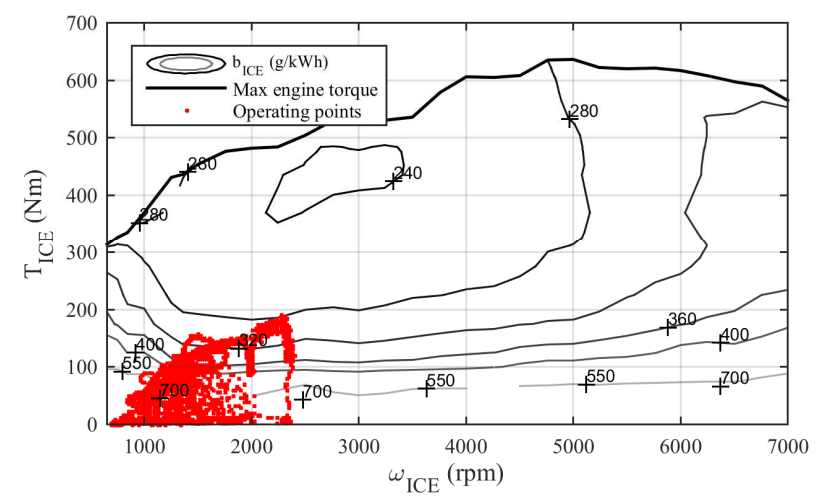

Fig. 8. Experimental $b_{I C E}\left(\omega_{I C E}, T_{I C E}\right)$ map for $\theta_{I C E}=90^{\circ} \mathrm{C}$, with indication of the ICE operating points during the NEDC.

\section{B. Simulation results}

The simulation model of Section V.A was used for the assessment of the fuel consumption of the same vehicle with the two transmissions solely driven by the ICE, along four driving cycles, namely: i) the New European Driving Cycle (NEDC); ii) the Federal Test Procedure 75 (FTP-75); iii) the Supplemental Federal Test Procedure (US06); and iv) the Worldwide harmonized Light vehicles Test Procedures for high power vehicles (WLTP $\mathrm{cl} 3$ ). The simulations consider the marginal vehicle mass difference associated with the HAMT and DCT, i.e., $\sim 70 \mathrm{~kg}$ of penalty for the HAMT, which depends on the size of the energy storage system.

The average fuel consumption per unit distance, the related $\mathrm{CO}_{2}$ emission per unit distance, and the $\mathrm{CO}_{2}$ emission reduction (in percentage) caused by the HAMT are reported in Fig. 9 for the selected driving cycles. The emission reduction of the HAMT ranges from $\sim 9.2 \%$ to $\sim 11.5 \%$. These significant values are achieved as the case study transmissions operate in their very low torque range during the driving schedules. In these conditions the gap among the DCT and HAMT efficiencies is particularly evident even at high temperatures.

During the analysis of the simulation results, it was verified that the difference in fuel consumption is caused by the reduction - by more than $40 \%$ - of the transmission power losses, while the difference in internal combustion engine efficiency related to the variation of the operating points of the ICE has a negligible effect. The transmission performance simulation in actual operating conditions - rather than the more conventional analysis based on the efficiency map comparison - is particularly relevant for the specific high performance 
vehicle, because its ICE and transmission normally work in a very limited torque and speed range. For example, the operating points of the ICE during the NEDC are included in Fig. 8. In such limited operating range the transmission efficiency can differ quite significantly from its average value for the whole range of possible operation. In fact, the drivetrain components of the specific vehicle are designed for maximum power and input torque levels in excess of $450 \mathrm{~kW}$ and 650 $\mathrm{Nm}$, respectively.

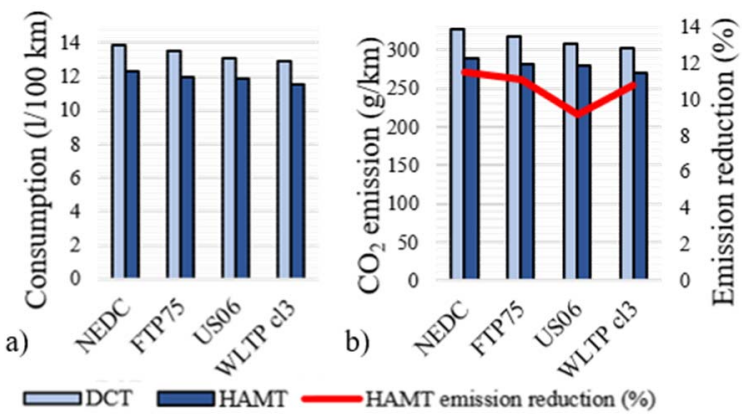

Fig. 9. Simulation results of the case study vehicle with the DCT and HAMT in ICE mode: a) fuel consumption per unit distance; b) $\mathrm{CO}_{2}$ emission per unit distance and $\mathrm{CO}_{2}$ emission reduction (in percentage) caused by the HAMT.

\section{CONCLUSION}

The paper described the layout of a novel hybridized automated manual transmission (HAMT) for high performance passenger cars. The HAMT provides a flexible hybrid electric drivetrain configuration, including fully electric capability. Moreover, the HAMT allows gearshifts with torque-fill on the ICE side of the transmission through the appropriate actuation of the electric motor, and reduces the power losses in its ICE mode with respect to the corresponding DCT. In this study the efficiencies of the specific production DCT and HAMT prototype were experimentally measured on a transmission test rig, and the resulting maps were used for vehicle simulations along a selection of driving cycles.

The main conclusions are:

- The experimental efficiency measurements of the two transmissions on a test rig show the higher efficiency of the HAMT in its ICE mode with respect to the DCT. In particular, the relative efficiency increase ranges between $\sim 5 \%$ and $\sim 13 \%$ at medium-high input torque values, depending on the selected gear and operating temperature.

- Larger efficiency improvements are achieved in the ICE mode of the HAMT at low torque demands, which are typical of the selected driving cycles. These benefits are mainly caused by the reduction of the power losses associated with the ancillary systems of the HAMT, which is characterized by the absence of a wet clutch pack.

- The efficiency of the fully electric mode of the HAMT in P3 configuration is higher than that of its P2 configuration, especially when the system is operating at high output speeds. The HAMT operation in the I EM gear is more efficient than that in the II EM gear, in the investigated torque range.

- In fully electric mode, on average the HAMT is marginally more efficient in traction than in regeneration, while the general trend of the efficiency maps is similar for the two cases.

- The fuel consumption reduction of the ICE mode of the HAMT along the selected driving cycles ranges from $\sim 9.2 \%$ to $\sim 11.5 \%$, with respect to the DCT. The significance of these values means that the appropriate energy efficiency-oriented design of the transmission system should become an important point in the industrial drivetrain development process.

Future work will focus on: i) the experimental assessment of the ICE mode and fully electric mode of the HAMT along driving cycles; and ii) the assessment and validation of the further fuel consumption reductions associated with the optimal control of the hybrid mode of the HAMT.

\section{REFERENCES}

[1] M. Kulkarni, T. Shim, and Y. Zhang, "Shift dynamics and control of dual-clutch transmissions," Mechanism and Machine Theory, vol. 42, no. 2, pp. 168-182, 2007.

[2] H. Webster, "A Fully Automatic Vehicle Transmission Using a Layshaft Type Gearbox," SAE Technical Paper 810104, SAE International, Warrendale, PA, USA, 1981.

[3] F. Vacca, S. De Pinto, A.E. Hartavi Karci, P. Gruber, F. Viotto, C.. Cavallino, J. Rossi, and A. Sorniotti, "On the energy efficiency of dual clutch transmissions and automated manual transmissions," Energies, vol. 10, 1562, 2017.

[4] Y. Yang, X. Hu, H. Pei, and Z. Peng, "Comparison of power-split and parallel hybrid powertrain architectures with a single electric machine: dynamic programming approach," Applied Energy, vol. 168, Supp. C, pp. 683-690, 2016.

[5] G. Hellenbroich, and V. Rosenburg, "FEV's new parallel hybrid transmission with single dry clutch and electric torque support," VDI Berichte, no. 2071, pp. 467-478, 2009.

[6] F. Falzoni, "Seven-Gear Gearbox for a Motorcar Double Clutch Transmission," U.S. Patent 7,562,598, 2009.

[7] A.M. Gavgani, T. Bingham, A. Sorniotti, J. Doherty, C. Cavallino, and M. Fracchia, "A parallel hybrid electric drivetrain layout with torque-fill capability," SAE International Journal of Passenger Cars - Mechanical Systems, vol. 8, no. 2, pp. 767-778, 2015.

[8] A.M. Gavgani, A. Sorniotti, J. Doherty, and C. Cavallino, "Optimal gearshift control for a novel hybrid electric drivetrain," Mechanism and Machine Theory, vol. 105, pp. 352-368, 2016.

[9] SAE Recommended Practice, "Manual transmission and transaxle efficiency and parasitic loss measurement," No. J2453, SAE International, Warrendale, PA, USA, 2011.

[10] A. Pennycott, L. De Novellis, P. Gruber, and A. Sorniotti, "Sources of power loss during torque-vectoring for fully electric vehicles," International Journal of Vehicle Design, vol. 67, no. 2, pp. 157-177, 2015.

[11] L. De Novellis, A. Sorniotti, and P. Gruber, "Wheel torque distribution criteria for electric vehicles with torque-vectoring differentials," IEEE Transactions on Vehicular Technology, vol. 63, no. 4, pp. 1593-1602, 2014.

[12] Environmental Protection Agency, "Greenhouse gas emissions from a typical passenger vehicle," EPA-420-F-14-040a, U.S. Environmental Protection Agency, Ann Arbor, MI, USA, 2014. 